\title{
SIGNAL PHOTOELECTRON YIELD DEPENDENCE ON THE X-RAY ANGLE OF INCIDENCE
}

\author{
I.S. Tilinin*, A. Jabloński, B. Lesiak-Orlowska \\ Institute of Physical Chemistry, Polish Academy of Sciences \\ Kasprzaka 44/52, 01-224 Warszawa, Poland
}

\begin{abstract}
The photoelectron emission from solids irradiated by X-rays was described by the analytical theory of electron transport and simulated by the Monte Carlo technique. The medium energy electron transport problem is treated by means of a Boltzmann type kinetic equation satisfying appropriate boundary conditions. The solution of the transport equation was obtained in the transport approximation based on the generalized radiative field similarity principle. Simple and reliable formalism was derived for both the differential and the total photoelectron yields. The dependence of the photoelectron yield on the X-ray incidence angle and the "flattening" effect of multiple elastic scattering on the angular distribution of electrons leaving the target are analysed in detail. The photoelectron yields and angular distributions calculated by the Monte Carlo algorithm, based on a realistic differential elastic scattering cross-section, are in good agreement with the results found from analytical theory. It is shown that main characteristics of the photoelectron emission are determined primarily by two parameters: the inelastic and the transport mean free paths.
\end{abstract}

PACS numbers: 79.60.-i, 72.10.Bg, 34.80.Bm

\section{Introduction}

When passing through matter, X-rays may ionize low lying atomic shells and create photoelectrons whose initial velocities are determined by the absorbed photon energy and the binding energy of the corresponding atomic shell. Some of those electrons, after travelling a certain path length, can reach a target surface and leave the irradiated sample. The photoelectrons escaping from the target without being scattered inelastically retain their initial kinetic energy and thereby carry direct information about chemical composition of top surface layers. Emission of signal photoelectrons has been a subject of intensive studies during the last decade [1-4] due to wide application of the X-ray photoelectron spectroscopy in

*On leave from Moscow Engineering Physics Institute, Kashirskoye sh., 31, Moscow 115409, Russia. 
surface analysis [5]. It has been realized that proper account of elastic scattering of electrons on their way out of the target would improve significantly quantification of experimental data concerning both the angular spectra and the escape probability as a function of the depth of origin of photoelectrons.

This paper is devoted to further studies of the signal photoelectron yield dependence on the basic electron transport characteristics as well as on the geometrical conditions.

\section{Theory}

Let a broad beam of X-rays be incident on a flat target at the polar angle $\theta$ counted from the surface normal. The effective escape depth of the photoelectrons leaving the target without being scattered inelastically is usually small compared with the absorption and the transport mean free paths of photons in the matter. Hence, the attenuation of the photon beam in the near surface region providing the major contribution to the photoelectron yield can be neglected. It follows from this that the initial angular distribution of signal electrons can be regarded as independent of depth. In the case of non-polarized radiation and randomly oriented atoms and molecules, the initial spectrum of photoelectrons normalized to unity is given by the expression [6]:

$$
f\left(\Omega_{0}, \Omega_{\gamma}\right)=(4 \pi)^{-1}\left\{1-(\beta / 4)\left[3\left(\Omega_{0} \Omega_{\gamma}\right)^{2}-1\right]\right\},
$$

where $\Omega_{0}$ and $\Omega_{\gamma}$ are the unit vectors along the directions of photoelectron emission from atoms and X-ray propagation respectively, and $\beta$ is the asymmetry parameter. To find the angular distribution of photoelectrons leaving the sample it is necessary to solve a kinetic equation with a boundary conditions implying that no electrons enter the target [3, 7]. Recently Werner and Tilinin [3] found an expression for the angular distribution of photoelectrons escaping from the target in the case of azimuthally symmetrical problem. Further generalization of their result for an arbitrary geometry leads to a formula for the differential photoelectron yield $Y(\Omega)$, where $Y(\Omega) \mathrm{d} \Omega$ describes the number of electrons emitted from a unit area of the surface in the elementary solid angle $\mathrm{d} \Omega$ along the direction of the unit vector $\Omega$. In the present paper, the attention is paid to analysis of the photoelectron angular spectrum with respect to the ernission polar angle $\alpha$ counted from the surface normal and the total photoelectron yield.

Taking into account the initial angular distribution of electrons (Eq. (1)) and integrating the differential photoelectron yield $Y(\Omega)$ over the azimuthal angle we arrive at the following formula for the photoelectron distribution with respect to the cosine of the emission angle $\mu=\cos \alpha$ :

$$
\begin{aligned}
Y(\mu) & =\left(\mu y_{0} / 2\right)\left[\frac{H(\mu, \omega)}{(1-\omega)^{1 / 2}}-\frac{\beta}{8}\left(3 \mu_{\gamma}^{2}-1\right)\left(3 \mu^{2}-1\right)\right. \\
& \left.+\frac{\omega \beta}{16} \int_{0}^{1} \frac{x H(x, \omega) H(\mu, \omega)}{x+\mu}\left(3 \mu_{\gamma}^{2}-1\right)\left(3 x^{2}-1\right) \mathrm{d} x\right] .
\end{aligned}
$$

In the latter expression $H(\mu, \omega)$ is the $H$-function of Chandrasekhar for an isotropically scattering medium [8], $\mu_{\gamma}=\cos \theta$, the parameter $y_{0}$ and the single scattering albedo $\omega$ are defined by

$$
y_{0}=n_{0} F \sigma_{\mathrm{ph}} \lambda_{\mathrm{tr}} \omega, \quad \omega=\lambda_{\mathrm{i}} \lambda_{\mathrm{tr}} /\left(\lambda_{\mathrm{i}}+\lambda_{\mathrm{tr}}\right)
$$


where $n_{0}$ is the atomic density, $\sigma_{\text {ph }}$ is the total photoelectric cross-section, $F$ is the incident photon flux, $\lambda_{\mathrm{i}}$ and $\lambda_{\mathrm{tr}}$ are the inelastic and the transport mean free path respectively. It should be emphasized that formula (2) is found in the transport approximation implying replacement of a real differential elastic scattering cross-section in the kinetic equation by an isotropic one being equal to the corresponding momenturn transfer cross-section. The validity of the transport approximation is discussed in detail elsewhere $[3,7]$.

The analysis shows that for the relatively small values of single scattering albedo $\omega<0.5$, typical of medium energy range electrons, the contribution of the integral term in the right side of Eq. (2) to the differential yield is usually small compared with that of the first term, and does not exceed a few percent. Therefore, the integral term may be omitted in practical assessments and we obtain a simple expression for the angular spectrurn of photoelectrons leaving the target

$$
Y(\mu) \sim\left(\mu y_{0} / 2\right)\left[\frac{H(\mu, \omega)}{(1-\omega)^{1 / 2}}-\frac{\beta}{8}\left(3 \mu_{\gamma}^{2}-1\right)\left(3 \mu^{2}-1\right)\right] .
$$

In the limiting case of strong absorption $\lambda_{\mathrm{i}} \ll \lambda_{\mathrm{tr}}$ formula (3) reduces to the result of the straight line approximation

$$
Y(\mu) \sim \mu\left(\lambda_{\mathrm{i}} / l_{\mathrm{a}}\right) f\left(\mu, \mu_{\gamma}\right),
$$

where $f\left(\mu, \mu_{\gamma}\right)$ is the initial angular spectrum integrated over the azimuthal angle, and $l_{\mathrm{a}}=\left(n_{0} \sigma_{\mathrm{ph}}\right)^{-1}$ is the absorption mean free path of photons in the solid. In the opposite limiting case of intensive elastic scattering, $\lambda_{\mathrm{i}} \gg \lambda_{\mathrm{tr}}$, we have

$$
Y(\mu) \sim \mu\left(\lambda_{\mathrm{i}} \lambda_{\mathrm{tr}} / l_{\mathrm{a}}\right)^{1 / 2} H(\mu, 1) .
$$

The latter result is derived by neglecting all the terms but the first one on the right side of Eq. (2) and by putting $1-\omega \sim \lambda_{\text {tr }} / \lambda_{\mathrm{i}}$. From (4), it follows that when elastic scattering is ignored, the angular distribution of electrons leaving the sample becomes proportional to their initial spectrum $f\left(\mu, \mu_{\gamma}\right)$. On the contrary, if particles experience intensive multiple elastic scattering they completely lose information about the initial direction of motion (see Eq. (5)). Generally, the differential yield $Y(\mu)$ may be presented as the sum of two functions, one of which is proportional to the initial distribution $f\left(\mu, \mu_{\gamma}\right)$ while the other is equal to $r(\mu)=\left(\mu y_{0} / 2\right)\left[H(\mu, \omega)(1-\omega)^{-1 / 2}-1\right]$. Obviously the function $r(\mu)$ describes the randornized fraction of the photoelectron yield. The effective escape depth of those electrons increases with augmenting the inelastic mean free path proportionally to $\left(\lambda_{\mathrm{i}} \lambda_{\mathrm{tr}}\right)^{1 / 2}$ which is clearly seen from Eq. (3). On the contrary, the particles whose angular distribution is directly proportional to the differential photoelectric cross-section escape from the target without being deflected at all. To be precise, they may suffer small angle scattering on their way out of the sample. In the transport approximation this effect is accounted for by replacing the elastic mean free path $\lambda_{\mathrm{e}}$ by the transport mean free path $\lambda_{\mathrm{tr}}$. For a sharply peaked forward differential elastic scattering cross-section the quantity $\lambda_{\mathrm{tr}} \gg \lambda_{\mathrm{e}}$. Hence we conclude that in the approach considered the group of undeflected electrons comprises both the electrons that do not experience elastic collisions at all and the particles that suffer small angle elastic scattering. In practically relevant cases the contributions of these two groups of electrons to the yield are of the same order of magnitude 
and the corresponding mean escape depths differ usually by several tens percent from each other.

The total yield of signal photoelectrons $y$ can be found by integrating the distribution $Y(\mu)$ over the $\mu$ variable. Performing the integration we find

$$
\begin{aligned}
y= & y_{0}\left\{\frac{\nu_{0}-1+\chi_{0}}{\omega}-\frac{\beta}{64}\left(3 \mu_{\gamma}^{2}-1\right)\right. \\
& \left.\times\left[1+2 \omega \int_{0}^{1} \int_{0}^{1} \frac{x H(x, \omega) H(\mu, \omega)\left(3 x^{2}-1\right)}{x+\mu} \mathrm{d} x \mathrm{~d} \mu\right]\right\} .
\end{aligned}
$$

Here $\nu_{0}$ is the positive root of the characteristic equation

$$
1=\left(\omega \nu_{0} / 2\right) \ln \left[\left(\nu_{0}+1\right) /\left(\nu_{0}-1\right)\right]
$$

and $\chi_{0}$ is the integral

$$
\chi_{0}=(1 / \pi) \int_{0}^{1} \chi(\mu) \mathrm{d} \mu
$$

where the function $\chi(\mu)$ is defined by

$$
\chi(\mu)=\left\{\begin{array}{cc}
\operatorname{arctg} x(\mu), & x>0, \\
\pi+\operatorname{arctg} x(\mu), & x<0,
\end{array}\right.
$$

and $x(\mu)=(\omega \mu \pi / 2)\{1-(\omega \mu / 2) \ln [(1+\mu) /(1-\mu)]\}^{-1}$.

It can be easily shown that the double integral in the right side of Eq. (6) is as a rule small compared with unity and may be often neglected. If this is the case we get for the total yield the simple formula

$$
y=y_{0}\left[\left(\nu_{0}-1+\chi_{0}\right) / \omega-(\beta / 64)\left(3 \mu_{\gamma}^{2}-1\right)\right] .
$$

The total yield varies slowly with the cosine of X-ray angle of incidence $\mu_{\gamma}$, and reaches its maximum value at $\mu_{\gamma}=0$. In the limiting case of strong absorption $\lambda_{\mathrm{i}} \ll \lambda_{\text {tr }}$ the elastic scattering effect is negligible and, as it is expected, formula (6) reduces to the result of the straight line approximation

$$
y=\left(y_{0} / 4\right)\left[1-(\beta / 16)\left(3 \mu_{\gamma}^{2}-1\right)\right] .
$$

In the opposite limiting case of weak absorption $\lambda_{\mathrm{i}} \gg \lambda_{\mathrm{tr}}$ the positive root of the characteristic equation becomes large compared with unity, $\nu_{0} \gg 1$. As a result the yield $y$ is mainly determined by diffusely scattered electrons and is almost independent of the $\mathrm{X}$-ray angle of incidence, $y \sim\left(\lambda_{\mathrm{i}} \lambda_{\mathrm{tr}} / 3 l_{\mathrm{a}}\right)^{1 / 2}$ in accordance with the recent finding [7].

On the basis of the developed approach, it is possible also to calculate the so-called partial yields $y^{+}$and $y^{-}$. The quantity $y^{+}$represents the total amount of photoelectrons emitted initially towards the surface and leaving the target. Correspondingly $y^{-}$equals the number of electrons that are ejected from atoms with the momenta directed inside the sample but escape from it due to elastic scattering. By definition we have $y=y^{+}+y^{-}$. The partial total yields are given by the expressions

$$
y^{+}=\left(y_{0} / 4\right)\left[2\left(\nu_{0}-1+\chi_{0}\right)+1-(\beta / 16)\left(3 \mu_{\gamma}^{2}-1\right)\right],
$$




$$
\begin{aligned}
y^{-}= & \left(y_{0} / 2\right)\left[\left(\nu_{0}-1+\chi_{0}\right)(2-\omega) / \omega-\frac{1}{2}\right. \\
& \left.+\frac{\beta}{8}\left(3 \mu_{\gamma}^{2}-1\right) \int_{0}^{1}\left[1-(1-\omega)^{1 / 2} H(x, \omega)\left(3 x^{2}-1\right)\right] x \mathrm{~d} x\right] .
\end{aligned}
$$

Owing to a small value of the integral in the square brackets of Eq. (13) the partial yield $y^{-}$varies extremely slowly with changing the X-ray angle of incidence. From the physical point of view this is consistent with the fact that the quantity $y^{-}$ comprises mostly the photoelectrons undergoing multiple elastic scattering and thereby being almost completely randomized. For rough assessments one can put $y^{-} \sim\left(y_{0} / 4 \omega\right)\left[2\left(\nu_{0}-1+\chi_{0}\right)(2-\omega)-\omega\right]$. The behaviour of the total yields $y^{+}$ and $y^{-}$in the corresponding limiting cases can be easily traced. We note only that the quantity $y^{-}$decreases rapidly with diminishing the single scattering albedo $\omega$.

\section{Comparison of analytical and Monte Carlo simulation results}

The Monte Carlo simulation technique represents not only an independent tool for photoelectron transport study but also provides an effective means of verification of analytical results in those cases when accuracy of an approximate solution is difficult to assess. In this report the Monte Carlo method was used to evaluate the photoelectron yields in a narrow and the $2 \pi$ solid angles for $\mathrm{Al}$,

\section{TABLE}

The dependence of the total photoelectron yield $y$ on the X-ray angle of incidence $\theta$. Calculations are made for the photoelectron energy $E=$ $689 \mathrm{eV}$, and the asymmetry parameter $\beta=2.0 . \mathrm{MC}-$ Monte Carlo

\begin{tabular}{|c|c|c|c|c|c|c|c|c|c|c|}
\hline \multirow{3}{*}{$\begin{array}{c}\theta \theta \\
{[\mathrm{deg}]}\end{array}$} & \multicolumn{10}{|c|}{ Total yield $y$ [electron $\cdot \mathrm{cm}^{-2} \cdot \mathrm{s}^{-1}$ ] } \\
\hline & \multicolumn{2}{|c|}{$\mathrm{Al}$} & \multicolumn{2}{|c|}{$\mathrm{Cu}$} & \multicolumn{2}{|c|}{$\mathrm{Ag}$} & \multicolumn{2}{|c|}{ W } & \multicolumn{2}{|c|}{$\mathrm{Pt}$} \\
\hline & $\mathrm{MC}$ & $\mathrm{AT}$ & $\mathrm{MC}$ & $\mathrm{A}^{\top} \mathrm{T}$ & $\mathrm{MC}$ & $\mathrm{AT}$ & $\mathrm{MC}$ & AT & $\mathrm{MC}$ & AT \\
\hline 0 & 2.85 & 2.76 & 2.37 & 2.34 & 2.04 & 2.04 & 1.93 & 1.91 & 1.91 & 1.88 \\
\hline 10 & 2.88 & 2.79 & 2.40 & 2.36 & 2.05 & 2.06 & 1.96 & 1.92 & 1.92 & 1.90 \\
\hline 20 & 2.98 & 2.91 & 2.45 & 2.43 & 2.11 & 2.12 & 2.00 & 1.97 & 1.97 & 1.95 \\
\hline 30 & 3.11 & 3.06 & 2.55 & 2.52 & 2.20 & 2.20 & 2.07 & 2.05 & 2.05 & 2.02 \\
\hline 40 & 3.29 & 3.25 & 2.67 & 2.64 & 2.32 & 2.30 & 2.16 & 2.14 & 2.13 & 2.10 \\
\hline 50 & 3.48 & 3.45 & 2.79 & 2.77 & 2.44 & 2.41 & 2.27 & 2.25 & 2.23 & 2.22 \\
\hline 60 & 3.67 & 3.64 & 2.91 & 2.89 & 2.55 & 2.54 & 2.36 & 2.34 & 2.30 & 2.30 \\
\hline 70 & 3.79 & 3.80 & 3.01 & 2.99 & 2.63 & 2.63 & 2.43 & 2.42 & 2.38 & 2.38 \\
\hline 80 & 3.88 & 3.89 & 3.05 & 3.06 & 2.71 & 2.69 & 2.48 & 2.47 & 2.42 & 2.43 \\
\hline 90 & - & 3.90 & - & 3.07 & - & 2.71 & - & 2.48 & - & 2.45 \\
\hline
\end{tabular}
simulation data, AT - analytical theory (Eq. (6)). 


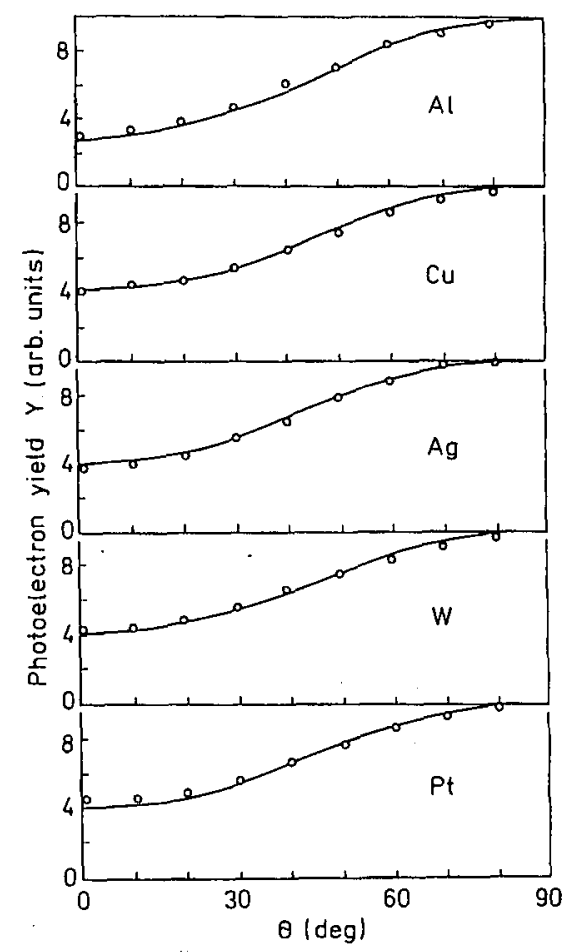

Fig. 1. The dependence of the photoelectron yield $Y$ collected in the narrow solid angle (the half cone acceptance angle $\Delta \alpha=10^{\circ}$ ) along the surface normal on the X-ray angle of incidence $\theta$ for the $\mathrm{Mn} 2 p_{3 / 2}$ line and the $\mathrm{Al}, \mathrm{Cu}, \mathrm{Ag}, \mathrm{W}$ and Pt targets. The photoelectron energy $E=613 \mathrm{eV}$, the asymmetry parameter $\beta=1.47$. Open circles Monte Carlo simnlation data, solid curves - analytical theory.

$\mathrm{Cu}, \mathrm{Ag}, \mathrm{W}$ and Pt targets and Ti $2 s, \mathrm{Mn} 2 p_{3 / 2}, \mathrm{Al} 2 p, \mathrm{Au} 4 f_{7 / 2}$ photoelectron lines corresponding to the electron initial energies of $689,613,1169$ and $1180 \mathrm{eV}$ respectively. Detailed description of the Monte Carlo procedure may be found elsewhere [9]. In Fig. 1 the photoelectron yield $Y$ collected in the narrow solid angle along the surface normal (half cone acceptance angle $\Delta \alpha=10^{\circ}$ ) is displayed as the function of X-ray angle of incidence $\theta$. The photoelectron energy is $613 \mathrm{eV}$ and the asymmetry parameter $\beta=1.47$.The solid lines are analytical results calculated by integrating expression (2) over $\mu$ from $\mu_{1}=\cos 10^{\circ}$ to $\mu_{2}=1$, while the open circles are the Monte Carlo data found for a realistic differential elastic scattering cross-section. It should be stressed that the analytical and Monte Carlo results were obtained for the same photoelectron source power. This made possible to cormpare the data found by the two approaches in absolute units. Largest discrepancies between the analytical and Monte Carlo results are observed for the normal incidence of X-rays and reach about $10 \%$. In other cases they do not exceed a few percent. In Table the total yields calculated for the $\mathrm{Ti} 2 s$ line are shown for different $\mathrm{X}$-ray angles of incidence. The agreement between the analytical theory and the Monte Carlo data is excellent: the discrepancies are smaller than $1 \%$ and 
do not exceed a statistical error of Monte Carlo calculations. Similar results were found for other photoelectron lines.

Good agreement between the theory based on the transport approxirnation and the Monte Carlo data indicates that the former can be effectively used in practical assessments of photoemission characteristics. The elastic scattering effect can be described with sufficient accuracy by the only parameter, the transport mean free path $\lambda_{\text {tr }}$.

\section{Acknowledgment}

This work has been supported by the grant 224669102 of the State Committee for Scientific Research (Republic of Poland).

\section{References}

[1] O.A. Basch nko, G.V. Machavariani, V.I. Nefedov, J. Electron Spectrosc. Relat. Phenom. 42, 235 (1984).

[2] A. Jabłoński, M.F. Ebel, Surf. Interface Anal. 6, 21 (1984).

[3] W.M.S. Werner, I.S. Tilinin, Appl. Surf. Sci. 70/71, 29 (1993).

[4] A. Jabłoniski, J. Zemek, Phys. Rev. B 48, 4799 (1993).

[5] Practical Surface Analysis, 2nd ed., Eds. D. Briggs, M.P. Seah, Vol. 1, Wiley, Salle+Sauerlander, Chichester 1990.

[6] R.F. Reilman, A. Msezane, S.T. Manson, J. Electron Spectrosc. Relat. Phenom. 8, 289 (1976).

[7] I.S. Tilinin, W.S.M. Werner, Phys. Rev. B 46, 13739 (1992).

[8] S. Chandrasekhar, Radiative Transfer, 1st ed., Clarendon Press, Oxford 1950, p. 6.

[9] A. Jabłoński, H.S. Hansen, C. Jansson, S. Tougaard, Phys. Rev. B 45, 3694 (1992). 